\title{
Os efeitos do método Pilates aplicado à pacientes de fisioterapia: uma revisão integrativa
}

\author{
The effects of the Pilates method applied to physiotherapy patients: an integrative review \\ Los efectos del método Pilates aplicado a pacientes de fisioterapia: una revisión \\ integradora
}

Camila de Souza Lopes ${ }^{1 *}$, Marcos Antônio Nunes Araújo.

\begin{abstract}
RESUMO
Objetivo: Revisar os efeitos benéficos do Método Pilates em pacientes de Fisioterapia, associado ou não a outras técnicas. Métodos: Estudo de revisão integrativa, realizado nas bases de dados Scielo, Medline e Biblioteca Virtual em Saúde entre os anos de 2014 a 2020, no qual totalizaram 20 estudos. Os descritores utilizados foram: "Pilates", "Terapia por Exercício" e "Exercício de Reabilitação". Os trabalhos selecionados foram os que exploravam o potencial do Método Pilates em oferecer benefícios à saúde do paciente, promovendo maior qualidade de vida. Resultados: Os resultados são significativos para pacientes hipertensos, na melhora no controle de glicemia de diabéticos na correção postural, função física e na redução da fadiga de pacientes com esclerose múltipla. E na redução de dores musculares, capacidade funcional e qualidade do sono e no tratamento da lombalgia com melhoria da postura e reequilíbrio da musculatura. Considerações finais: $O$ Pilates é um método utilizado como forma de tratamento por fisioterapeutas e dependendo do objetivo do tratamento, tem mostrado resultados positivos para diversas patologias e pode ser utilizado em pessoas de todas as idades.
\end{abstract}

Palavras-chave: Terapia por exercício, Pilates, Exercício de reabilitação.

\section{ABSTRACT}

Objective: Review the beneficial effects of the Pilates Method in Physiotherapy patients, associated or not with other techniques. Methods: An integrative review study carried out in the Scielo, Medline and Virtual Health Library databases between the years 2014 to 2020, in which 20 studies totaled. The descriptors used were: "Pilates", "Exercise Therapy" and "Rehabilitation Exercise". The selected works were those that explored the potential of the Pilates Method, which can offer benefits to the patient's health, promoting greater quality of life. Results: The results are significant for hypertensive patients, in improving the glycemic control of diabetics in postural correction, physical function and in reducing fatigue in patients with multiple sclerosis. And, in the reduction of muscle pain, functional capacity and quality of sleep and in the treatment of low back pain with improved posture and rebalancing of the muscles. Final considerations: Pilates is a method used as a form of treatment by physiotherapists and depending on the purpose of the treatment, it has shown positive results for several pathologies and can be used in people of all ages.

Key words: Exercise therapy, Pilates, Rehabilitation exercise.

\section{RESUMEN}

Objetivo: Revisar los efectos beneficiosos del método Pilates en pacientes de fisioterapia, asociados o no con otras técnicas. Métodos: Un estudio de revisión integral llevado a cabo en las bases de datos de Scielo, Medline y Virtual Health Library entre los años 2014 a 2020, en el que totalizaron 20 estudios. Los descriptores utilizados fueron: "Pilates", "Terapia de ejercicio" y "Ejercicio de rehabilitación". Los trabajos seleccionados fueron aquellos que exploraron el potencial del Método Pilates, que puede ofrecer beneficios para la salud del paciente, promoviendo una mayor calidad de vida. Resultados: Los resultados son significativos para pacientes hipertensos, ya que mejoran el control glucémico de los diabéticos en la corrección postural, la función física y reducen la fatiga en pacientes con esclerosis múltiple. $Y$ en la reducción del dolor muscular, la capacidad funcional y la calidad del sueño y en el tratamiento del dolor lumbar con una mejor postura y reequilibrio de los músculos. Consideraciones finales: Pilates es un método utilizado como forma de tratamiento por fisioterapeutas y, según el propósito del tratamiento, ha mostrado resultados positivos para varias patologías y puede usarse en personas de todas las edades.

Palabras clave: Terapia de ejercicio, Pilates, Ejercicio de rehabilitación.

1Universidade Estadual do Mato Grosso do Sul (UEMS), Dourados - MS. *E-mail: camiladesouzalopes@gmail.com SUBMETIDO EM: 3/2020 


\section{INTRODUÇÃO}

Quando criança, Joseph Hubertus Pilates nascido em Dusseldorf, cidade no oeste da Alemanha em 1880, foi acometido com febre reumática, asma e raquitismo. Por isso, para obter qualidade de vida para conviver como as outras crianças da época, foi necessário desenvolver e trabalhar fisicamente seu corpo. Com exercícios disciplinados e constantes, ele conseguiu atingir uma musculatura tão definida que serviu como modelo para atlas anatômicos. Na fase adulta, durante a primeira guerra mundial, foi o período em que fortaleceu mais seus conhecimentos sobre condicionamento físico (LIPOSCKI DB, et al., 2016).

Ainda em tempo de guerra, o criador do método, se dedicou a auxiliar na recuperação de soldados feridos, período em que apresentou os primeiros exercícios envolvendo alguns objetos para auxiliar seu trabalho, quais foram: molas, cordas e polias. Tais objetos foram usados nas camas dos hospitais para promoção da força, flexibilidade, resistência e tônus muscular com os pacientes debilitados. Tal experiência durante o período da guerra foi essencial para o nascimento do método e aprimoramento de seus estudos e conhecimentos (LIPOSCKI DB, et al., 2016).

Os princípios tradicionais do método são seis. O primeiro é a centralização que é o aperto do centro muscular do corpo ou casa de força, localizado entre o assoalho pélvico e a caixa torácica durante o exercício. O segundo é a concentração que é a necessária atenção cognitiva para realizar o exercício. $O$ terceiro é controle ou a gestão rigorosa da postura e movimento durante o exercício. O quarto é precisão da técnica do exercício. O quinto é a fluidez, ou seja, a transição suave de movimentos dentro da sequência de exercícios. O sexto princípio é a respiração para mover o ar para dentro e para fora dos pulmões em coordenação com o exercício (WELLS C, et al., 2012).

Os exercícios de Pilates diversificaram em extensão e uso em diferentes contextos. A extensão está relacionada às modificações nos exercícios para atender as diferentes necessidades de pacientes. Os autores afirmam que a ampla disseminação, se deu também pela remoção das restrições da marca registrada sobre o uso e termo Pilates. (WELLS C, et al., 2012). Assim, na fisioterapia, o Método Pilates (MP) é utilizado para acompanhamento de mudanças relacionadas à recuperação de lesões ou algum tipo de enfermidade para melhoria das condições físicas. Todavia, na reabilitação, é importante o conhecimento de suas aplicações, contraindicações, forma de utilização, além de oferecer ao paciente a técnica adequada às alterações apresentadas por ele. Ou seja, os exercícios são inseridos de acordo com a característica do paciente, respeitando as dificuldades individuais. Dessa forma, os exercícios de Pilates destinam-se a aprimorar a flexibilidade geral do corpo (VAZ RA, et al., 2012).

A maioria dos exercícios de Pilates conta com acessórios equipamentos que auxiliam na realização dos movimentos. Dentre os equipamentos estão o Cadillac, Ladder Barrel, Prancha de Molas, Reformer, Reformer Torre, Step Chair e Wall Unit e entre os acessórios estão a: Bola suíça, Faixa Elástica, Anel de pilates e o bozu. Esses aparelhos e acessórios ajudam na redução dos riscos inerentes aos comprometimentos secundários, ajuda na manutenção da integridade dos movimentos e mobilidade articular de áreas comprometidas, com otimização dos movimentos (SULLIVAN SB, et al., 2004).

Em virtudes dos fatos mencionados, o objetivo desse artigo foi realizar uma revisão integrativa sobre os efeitos benéficos do MP em pacientes de Fisioterapia com diferentes tipos de patologias, associado ou não a outras técnicas.

\section{MÉTODOS}

Nessa revisão integrativa o método empregado busca alcançar informações e assim constituir um leque de conhecimentos para responder a seguinte pergunta: Quais os benefícios do MP para pacientes de fisioterapia com diferentes tipos de patologias associado ou não a outras técnicas? Para tanto, as etapas elaboradas a partir da seleção do tema foram: questão da pesquisa, estabelecimentos dos critérios de inclusão e exclusão, pesquisa na literatura sobre o tema, estabelecimento das informações a serem extraídas dos artigos selecionados, categorização dos estudos, avaliação e resultados da revisão. A coleta de dados foi realizada em fontes secundárias por meio de levantamento de artigos na literatura, tendo como base de dados Scientific Electronic Library Online (Scielo), Sistema Online de Busca e Análise de Literatura 
Médica (Medline) e Biblioteca Virtual em Saúde (BVS), entre os anos de 2014 a 2020. Na busca dos artigos foram utilizados os descritores "Método Pilates", "Terapia por Exercício" e "Exercício de Reabilitação" utilizando o operador "e". Os critérios estabelecidos estão demonstrados conforme Figura 1.

Figura 1 - Critérios de Inclusão e Exclusão estabelecidos para busca de artigos nas bases de dados.

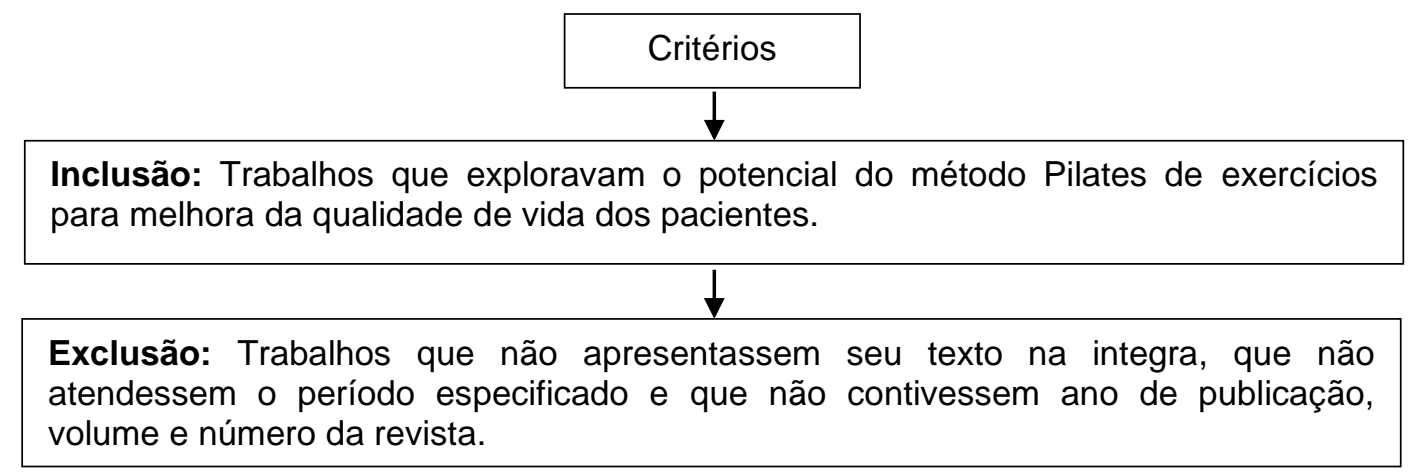

Fonte: Lopes CS e Araújo MAN, 2020.

\section{RESULTADOS E DISCUSSÃO}

Os resultados da pesquisa nos bancos de dados com a utilizando dos descritores resultou em um número de 28 artigos. Desse total foram excluídos oito artigos que não atendiam aos critérios estabelecidos nessa pesquisa. Assim, os artigos selecionados foram relacionados por temática e quantificados conforme demonstrado na Tabela 1. As publicações relacionadas com as capacidades físicas, dores musculares e lombares foram as mais evidentes na seleção das publicações. Em um percentual menor foram as publicações sobre doenças neurológicas, dores articulares e estados de humor.

Tabela 1 - Temas dos artigos relacionados com o MP.

\begin{tabular}{lc}
\hline Temas & $\mathbf{N}^{\circ}$ de Artigos \\
\hline Síndromes metabólicas & 2 \\
Doenças neurológicas & 1 \\
Dores musculares & 4 \\
Dores lombares & 4 \\
Dores articulares & 2 \\
Capacidades físicas & 5 \\
Estados de humor & 2 \\
\hline
\end{tabular}

Fonte: Lopes CS e Araújo MAN, 2020.

A Tabela 2 apresenta as publicações selecionadas que relatam os efeitos benéficos do MP em pacientes de fisioterapia relacionadas com síndromes metabólicas foram de 10\%; com doenças neurológicas 5\%; dores musculares $20 \%$; dores lombares $20 \%$; dores articulares $10 \%$. Assim também, o percentual de artigos relacionados às capacidades físicas foi de $25 \%$ e estados de humor resultaram em $10 \%$.

Tabela 2 - Tipos de estudos dos artigos selecionados.

\begin{tabular}{lc}
\hline Tipos de Estudo & Quantidade \\
\hline Revisão Sistemática & 4 \\
Revisão da Literatura & 7 \\
Revisão Narrativa & 1 \\
Estudo de Caso & 4 \\
Revisão Integrativa & 3 \\
Revisão Crítica & 1 \\
\hline Total & 20 \\
\hline
\end{tabular}

Fonte: Lopes CS e Araújo MAN, 2020.

Dos artigos selecionados para esse estudo, o Quadro 1 expõe as características das publicações, segundo o ano de publicação, autor, revista, objetivos do estudo e principais resultados. 
Quadro 1 - Publicações relacionadas aos benefícios do Método Pilates em Pacientes de Fisioterapia.

\begin{tabular}{|c|c|c|c|c|c|}
\hline Trabalho & Ano & Autor & Revista & Objetivos & Principais Resultados \\
\hline T1 & 2020 & $\begin{array}{l}\text { CORDEIRO } \\
\text { AL, et al. }\end{array}$ & $\begin{array}{l}\text { Pesquisa em } \\
\text { Fisioterapia. }\end{array}$ & $\begin{array}{l}\text { Revisar sistematicamente o efeito do } \\
\text { Pilates em pacientes com esclerose } \\
\text { múltipla. }\end{array}$ & $\begin{array}{l}\text { O MP apresentou resultados positivos na melhoria dos } \\
\text { padrões funcionais, força muscular, fadiga e equilíbrio, sendo } \\
\text { eficiente para o tratamento de pessoas com esclerose } \\
\text { múltipla. }\end{array}$ \\
\hline T2 & 2020 & $\begin{array}{l}\text { COSTA JCB, } \\
\text { et al. }\end{array}$ & Ciência Atual. & $\begin{array}{l}\text { Analisar os benefícios do Método } \\
\text { Pilates sobre os sintomas da } \\
\text { síndrome da Fibromialgia através de } \\
\text { uma revisão bibliográfica. }\end{array}$ & $\begin{array}{l}\text { O MP melhorou a sintomatologia de pacientes com } \\
\text { Fibromialgia, na melhoria da capacidade funcional, qualidade } \\
\text { do sono, diminuição da dor, do tensionamento muscular, } \\
\text { aumento de flexibilidade, melhora da capacidade respiratória } \\
\text { e diminuição da ansiedade. }\end{array}$ \\
\hline T3 & 2019 & $\begin{array}{l}\text { SILVA RBF, } \\
\text { et al. }\end{array}$ & Fisioterapia Brasil. & $\begin{array}{l}\text { Verificar os benefícios do Pilates para } \\
\text { sedentários, idosos e pacientes com } \\
\text { doenças cardiometabólicas. }\end{array}$ & $\begin{array}{l}\text { O MP, praticado regularmente e por longo tempo, reduziu } \\
\text { significativamente os níveis de ansiedade de sedentários. } \\
\text { Nos idosos, houve melhora no nível de estresse e redução de } \\
\text { riscos de quedas. Em pacientes com doenças } \\
\text { cardiometabólicas, houve redução da pressão arterial } \\
\text { sistólica e diastólica. }\end{array}$ \\
\hline T4 & 2020 & $\begin{array}{l}\text { FLEMING KM, } \\
\text { et al. }\end{array}$ & $\begin{array}{l}\text { Complementary } \\
\text { Therapies in } \\
\text { Medicine }\end{array}$ & $\begin{array}{l}\text { Examinar os efeitos agudos do } \\
\text { Pilates nos estados de humor de } \\
\text { jovens e adultos do sexo masculino. }\end{array}$ & $\begin{array}{l}\text { O MP reduziu a ansiedade do estado, os sentimentos de } \\
\text { fadiga e aumentou a sensação de energia nos pesquisados. }\end{array}$ \\
\hline T5 & 2019 & $\begin{array}{l}\text { ANTUNES } \\
\text { MD, et al. }\end{array}$ & $\begin{array}{l}\text { Rede de } \\
\text { Cuidados em } \\
\text { Saúde }\end{array}$ & $\begin{array}{l}\text { Verificar as contribuições do Método } \\
\text { Pilates e das orientações posturais } \\
\text { na dor, na qualidade de vida e na } \\
\text { sobrecarga de cuidadores de } \\
\text { pacientes neurológicos. }\end{array}$ & $\begin{array}{l}\text { O MP proporcionou a redução da dor e da sobrecarga de } \\
\text { cuidadores de pacientes neurológicos. Em relação à } \\
\text { qualidade de vida, os resultados mais favoráveis foram } \\
\text { observados nos domínios capacidade funcionais, limitação } \\
\text { por aspectos físicos, estado geral da saúde e saúde mental. }\end{array}$ \\
\hline T6 & 2019 & $\begin{array}{l}\text { COIMBRA } \\
\text { CMS, et al. }\end{array}$ & $\begin{array}{l}\text { Brazilian Jornal of } \\
\text { Development. }\end{array}$ & $\begin{array}{l}\text { Verificar os benefícios do Método } \\
\text { Pilates na flexibilidade de idosos. }\end{array}$ & $\begin{array}{l}\text { O MP contribuiu positivamente no equilíbrio, postura, } \\
\text { mobilidade e flexibilidade, sendo imprescindível para } \\
\text { autonomia e saúde do idoso. }\end{array}$ \\
\hline T7 & 2019 & $\begin{array}{l}\text { TORCINELLI } \\
\text { DL, et al. }\end{array}$ & Fisioterapia Brasil. & $\begin{array}{l}\text { Verificar se há influência do método } \\
\text { Pilates sobre a força muscular } \\
\text { respiratória, expansibilidade torácica } \\
\text { e pico de fluxo expiratório em } \\
\text { indivíduos tabagistas. }\end{array}$ & $\begin{array}{l}\text { O MP pode promover aumento da pressão inspiratória } \\
\text { máxima e do pico de fluxo expiratório. Porém, não há } \\
\text { influência do método sobre a amplitude de movimento da } \\
\text { caixa torácica. }\end{array}$ \\
\hline
\end{tabular}

REAS/EJCH | Vol.Sup.n.50 | e3307 | DOI: https://doi.org/10.25248/reas.e3307.2020 Página 4 de 12 


\begin{tabular}{|c|c|c|c|c|c|}
\hline Trabalho & Ano & Autor & Revista & Objetivos & Principais Resultados \\
\hline T8 & 2019 & $\begin{array}{l}\text { COSTA LPP, } \\
\text { et al. }\end{array}$ & $\begin{array}{l}\text { Ciência e } \\
\text { Movimento. }\end{array}$ & $\begin{array}{l}\text { Levantar, na literatura atual, estudos } \\
\text { relevantes que identifiquem as } \\
\text { possíveis respostas hemodinâmicas } \\
\text { encontradas em indivíduos } \\
\text { hipertensos praticantes do Método } \\
\text { Pilates. }\end{array}$ & $\begin{array}{l}\text { O MP apresentou resultados positivos na frequência cardíaca } \\
\text { (FC), pressão arterial (PA) e no duplo-produto (DP) em } \\
\text { comparação ao grupo controle. Os resultados das variáveis } \\
\text { hemodinâmicas nos levam a considerar plausível a utilização } \\
\text { do Pilates em pacientes hipertensos. }\end{array}$ \\
\hline T9 & 2018 & $\begin{array}{l}\text { CRUZ GL, et } \\
\text { al. }\end{array}$ & Faipe. & $\begin{array}{l}\text { Averiguar o Método Pilates como } \\
\text { benefício para os Portadores da } \\
\text { Síndrome da Fibromialgia, através de } \\
\text { um estudo de revisão bibliográfica } \\
\text { baseado em artigos científicos. }\end{array}$ & $\begin{array}{l}\text { O MP reduziu a tensão muscular geral, aliviou a dor da } \\
\text { fibromialgia generalizada, concentrando-se no fortalecimento } \\
\text { suave e baixo movimento de impacto. }\end{array}$ \\
\hline $\mathrm{T} 10$ & 2018 & $\begin{array}{l}\text { OSTERNO } \\
\text { MCR, et al. }\end{array}$ & Fisioterapia Ser. & $\begin{array}{l}\text { Analisar os benefícios do Pilates no } \\
\text { tratamento da artrose através de uma } \\
\text { revisão integrativa. }\end{array}$ & $\begin{array}{l}\text { O MP e sua prática é um excelente aliado na reabilitação de } \\
\text { pessoas idosas que se recuperam de traumas ou cirurgias e } \\
\text { melhoras do desenvolvimento funcional. }\end{array}$ \\
\hline $\mathrm{T} 11$ & 2015 & $\begin{array}{l}\text { JESUS, LT, } \\
\text { et al. }\end{array}$ & $\begin{array}{l}\text { Fisioterapia e } \\
\text { Pesquisa }\end{array}$ & $\begin{array}{l}\text { Avaliar a influência do método Pilates } \\
\text { sobre a função pulmonar, mobilidade } \\
\text { toracoabdominal, força muscular } \\
\text { respiratória e características } \\
\text { antropométricas em mulheres } \\
\text { saudáveis. }\end{array}$ & $\begin{array}{l}\text { No Grupo Pilates houve aumento significativo da atividade } \\
\text { física de lazer e no total do questionário de atividade física, } \\
\text { aumento da mobilidade nos três níveis (axilar, xifoidiano e } \\
\text { abdominal), da força muscular respiratória, tanto inspiratória } \\
\text { como expiratória, bem como redução significativa da } \\
\text { circunferência da cintura. }\end{array}$ \\
\hline $\mathrm{T} 12$ & 2017 & $\begin{array}{l}\text { POLETTO R, } \\
\text { et al. }\end{array}$ & $\begin{array}{l}\text { Saúde e } \\
\text { Desenvolv. } \\
\text { Humano. }\end{array}$ & $\begin{array}{l}\text { Revisar achados anteriores quanto } \\
\text { aos efeitos da prática do Método } \\
\text { Pilates por atletas, sejam eles } \\
\text { amadores ou não. }\end{array}$ & $\begin{array}{l}\text { O MP trouxe benefícios no desempenho esportivo, } \\
\text { coordenação, flexibilidade, força muscular e postura. }\end{array}$ \\
\hline T13 & 2017 & $\begin{array}{l}\text { SOUZA AML, } \\
\text { et al. }\end{array}$ & $\begin{array}{l}\text { Científica do } \\
\text { Centro } \\
\text { Universitário de } \\
\text { Jales. }\end{array}$ & $\begin{array}{l}\text { Verificar como o tratamento com o } \\
\text { método Pilates é eficaz em bailarinos } \\
\text { com dor lombar e desequilíbrio } \\
\text { muscular. }\end{array}$ & $\begin{array}{l}\text { O MP ajudou no alongamento e relaxamento dos músculos } \\
\text { encurtados e fortalecimento daqueles enfraquecidos e na } \\
\text { redução dos desequilíbrios musculares que ocorre devido à } \\
\text { dança. }\end{array}$ \\
\hline $\mathrm{T} 14$ & 2017 & $\begin{array}{l}\text { MOREIRA F, } \\
\text { et al. }\end{array}$ & Uningá. & $\begin{array}{l}\text { Avaliar os benefícios do método } \\
\text { Pilates em indivíduos com hipercifose } \\
\text { na coluna torácica. }\end{array}$ & $\begin{array}{l}\text { O MP proporcionou o equilíbrio entre força e flexibilidade } \\
\text { muscular por meio do desenvolvimento da consciência } \\
\text { corporal em pacientes com hipercifose na coluna torácica. }\end{array}$ \\
\hline
\end{tabular}

REAS/EJCH | Vol.Sup.n.50 | e3307 | DOI: https://doi.org/10.25248/reas.e3307.2020 Página 5 de 12 


\begin{tabular}{|c|c|c|c|c|c|}
\hline Trabalho & Ano & Autor & Revista & Objetivos & Principais Resultados \\
\hline T15 & 2016 & $\begin{array}{l}\text { FERREIRA } \\
\text { TN, et al. }\end{array}$ & $\begin{array}{l}\text { Saúde \& Ciência } \\
\text { em Ação. }\end{array}$ & $\begin{array}{l}\text { Verificar os efeitos do método Pilates } \\
\text { em pacientes com lombalgia. }\end{array}$ & $\begin{array}{l}\text { O MP proporcionou o fortalecimento da musculatura do } \\
\text { tronco e abdômen. }\end{array}$ \\
\hline T16 & 2016 & $\begin{array}{l}\text { COSTA } \\
\text { LMR, et al. }\end{array}$ & $\begin{array}{l}\text { Brasileira de } \\
\text { Geriatria e } \\
\text { Gerontologia. }\end{array}$ & $\begin{array}{l}\text { Revisar os efeitos do Método Pilates } \\
\text { em idosos. }\end{array}$ & O MP melhorou o equilíbrio e o risco de quedas nos idosos. \\
\hline $\mathrm{T} 17$ & 2016 & $\begin{array}{l}\text { AGUIAR SD, } \\
\text { et al. }\end{array}$ & $\begin{array}{l}\text { Conscientiae } \\
\text { Saúde }\end{array}$ & $\begin{array}{l}\text { Avaliar a eficácia do Método Pilates } \\
\text { para a melhora da dor em pacientes } \\
\text { com fibromialgia }\end{array}$ & Os efeitos do MP são positivos para redução da dor. \\
\hline T18 & 2015 & $\begin{array}{l}\text { FERREIRA } \\
\mathrm{GQ} \text {, et al. }\end{array}$ & $\begin{array}{l}\text { Visão } \\
\text { Universitária. }\end{array}$ & $\begin{array}{l}\text { Mostrar através de uma pesquisa de } \\
\text { revisão bibliográfica que o método } \\
\text { Pilates como tratamento é eficaz em } \\
\text { pacientes com dores na região } \\
\text { lombar. }\end{array}$ & $\begin{array}{l}\text { O MP é eficaz no tratamento da dor na região lombar, pois a } \\
\text { técnica trabalha com o alongamento e fortalecimento e } \\
\text { preconiza a respiração associada com a contração muscular } \\
\text { para um bom resultado. }\end{array}$ \\
\hline T19 & 2014 & $\begin{array}{l}\text { NASCIMENT } \\
\text { O SL, et al. }\end{array}$ & $\begin{array}{l}\text { Brasileira de } \\
\text { Ginecologia e } \\
\text { Obstetrícia. }\end{array}$ & $\begin{array}{l}\text { Disseminar informações entre } \\
\text { profissionais de saúde, que assistem } \\
\text { às gestantes no Brasil, das atuais } \\
\text { recomendações sobre exercício físico } \\
\text { durante a gestação, baseadas nas } \\
\text { melhores evidências científicas } \\
\text { disponíveis. }\end{array}$ & $\begin{array}{l}\text { O benefício do fortalecimento muscular permitiu melhoras na } \\
\text { postura, prevenção de quedas e traumas, bem como a } \\
\text { prevenção e de desconfortos musculoesqueléticos nas } \\
\text { gestantes. }\end{array}$ \\
\hline T20 & 2014 & $\begin{array}{l}\text { EVANGELIS } \\
\text { TA AL, et al. }\end{array}$ & Fisioterapia Brasil. & $\begin{array}{l}\text { Analisar a influência do método } \\
\text { Pilates nos estados de humor em } \\
\text { indivíduos fisicamente ativos. }\end{array}$ & $\begin{array}{l}\text { O MP contribuiu em reduções significativas dos estados de } \\
\text { humor com reduções significativas nos momentos de pré x } \\
\text { pós - sessão, na tensão, raiva e confusão de cada paciente. }\end{array}$ \\
\hline
\end{tabular}

Fonte: Lopes CS e Araújo MAN, 2020. 


\section{MP e Síndrome Metabólicas}

As síndromes metabólicas alteram o funcionamento geral do organismo, quer seja por alterações químicas ou pela celeridade que elas ocorrem. Anualmente o número de pessoas diagnosticadas com algum tipo de doença relacionado a essa síndrome tem aumentado. A obesidade, hipertensão arterial, alterações do colesterol, triglicérides e glicemia, são os principais vilões (SBEM, 2007).

A prática do exercício físico em pacientes com hipertensão, de forma segura, principalmente pelo constante trabalho respiratório realizado, pode estar associada à diminuição da tensão, na obtenção do relaxamento do organismo e redução da pressão arterial. Logo, a prática de exercício de resistência e força com o Pilates tende a aumentar a força de ejeção do sangue na parede vascular gerando oxido nítrico e proporcionado um efeito vasodilatador (JUNGES S, et al., 2015).

O estudo de Costa LPP, et al. (2019), apresentou resultados positivos para a frequência cardíaca (FC), pressão arterial (PA) e no duplo-produto (DP) de pacientes hipertensos aos quais analisaram que a prática de exercício físico, como o MP se insere como uma prática segura e eficaz para esses pacientes.

Da mesma forma, Silva RBF e Guerino MF (2019) constataram os benefícios e aplicabilidade do MP para melhorar a qualidade de vida, reduções estatisticamente significativas na pressão arterial sistólica e diastólica, índice de massa corporal, percentual de gordura, massa gorda, relação cintura-quadril e pressão arterial em grupos estudados, com impacto clinicamente relevante na morbidade por doenças cardiovasculares e cerebrovasculares com a utilização do MP.

A prática do exercício físico regular, como o Pilates, integra à prevenção e tratamento dos pacientes diabéticos por melhorar o controle da glicemia e reduzir a gordura do corpo do paciente. A regulação dos receptores de insulina torna as fibras musculares sensíveis à insulina reduzindo-a, fazendo com que haja a diminuição do risco de desenvolver outras doenças associadas. (SANTOS SN, et al., 2017).

Além disso, estudos reforçam a importância de programas de exercícios em longo prazo para o tratamento e prevenção da diabetes e suas complicações. Tais estudos indicam que a epidemia de diabetes está associada a níveis baixos de atividade física e prevalência de obesidade.

Além do mais, pesquisadores e cientistas concordam que a atividade física é importante na terapia de qualquer tipo de diabetes. Assim, o MP vem ganhando cada vez mais notoriedade em hospitais e clínicas especializadas e sua prática tem potencial para prevenir fatores de risco cardiometabolicos por conciliar exercício postural (ADA, 2009).

\section{MP e doenças neurológicas}

A esclerose múltipla é uma doença crônica e autoimune, embora, a causa da doença ainda seja desconhecida, ela tem sido foco de muitos estudos no mundo todo, o que têm possibilitado uma constante e significativa evolução na qualidade de vida dos pacientes (ABEM, 2016)

No estudo de revisão sistemática de Cordeiro AL, et al. (2020) com ensaios clínicos randomizados com MP aplicado a pacientes com esclerose múltipla, conclui-se que o MP, apresenta resultados positivos com a correção postural, o trabalho respiratório, de equilíbrio e propriocepção durante o exercício, auxiliam no desenvolvimento do controle motor e no fortalecimento dos músculos eretores e abdominais, auxiliando no tratamento de pacientes com Esclerose Múltipla que manifestam déficits tanto sensoriais quanto motores.

O MP é uma terapia viável para pessoas com esclerose múltipla que pode levar a melhorias em sua função física e pode ser útil para reduzir a fadiga auto percebida (DUFF WRD, et al., 2018). Também pode ser direcionado para programas de fisioterapia no desequilíbrio e perda de força, que são as incapacidades primárias em pacientes com esclerose múltipla, em decorrência do treinamento de fortalecimento e equilíbrio (GUCLU-GUNDUZ ARZU, et al., 2014).

Assim, os potenciais efeitos benéficos do Pilates não são significativamente maiores do que os derivados do desempenho de outras terapias físicas. No entanto, mais estudos são necessários para consolidar as evidências científicas existentes sobre os benefícios do MP nas doenças neurológicas. 


\section{MP e dores musculares}

A fibromialgia é uma dor muscular com condição para envolver o processamento aferente central desordenado. Dentre os principais sintomas da fibromialgia incluem dor multifocal, fadiga, distúrbios do sono e problemas cognitivos ou de memória (SMITH HS, et al., 2011).

O estudo de Aguiar SD, et al. (2016) em mulheres com a faixa etária de 40 a 65 anos com diagnóstico de fibromialgia, com característica da dor difusa, com duração maior que três meses, com quadro de alterações do sono e fadiga excessiva e sem conhecimento prévio do MP. A aplicação de exercícios, avaliação e reavaliação da intensidade da dor crônica pela escala Visual Analógica (EVA) e pelo aparelho Augômetro, utilizando um ângulo de aproximação de noventa graus entre a superfície de estimulação e o ponto estimulado.

A qualidade de vida foi avaliada através do Questionário sobre Impacto da Fibromialgia (QIF) e o Índice de Qualidade do sono de Pittsburgh (IQSP). Os autores concluíram que o MP mostrou ser eficaz na redução da dor na melhora do sono e impacto da fibromialgia em pacientes após quinze sessões de tratamento. Prontamente, os efeitos são positivos para redução da dor, melhora da capacidade funcional e qualidade do sono.

Assim também, no estudo Costa JCB, et al. (2020) sobre os benefícios do MP na fibromialgia, quando realizado de maneira regular e correto, melhora o condicionamento físico, mental e melhora a qualidade de vida dos pacientes. Também, contribui para o fortalecimento muscular, redução da tensão da musculatura, alívio da dor generalizada, melhora a flexibilidade. Todavia, há uma necessidade de esclarecimentos de como sistematizar os exercícios e movimentos indicados e contraindicados, assim como, mais análises dos diferentes efeitos que o método pode promover para os portadores da fibromialgia.

O MP possui exercícios e equipamentos versáteis para favorecer no tratamento dos casos de pacientes com Fibromialgia, visto que é uma patologia em que o paciente sente dores nos quatro quadrantes do corpo. Logo, o alongamento e fortalecimento muscular trabalhado de forma holística e relaxante fazem com que os movimentos sejam realizados como forma de prevenção de lesões musculoesqueléticas e redução sintomática da patologia (CRUZ GL, et al, 2018).

Jesus LT, et al. (2015) em um ensaio clínico não randomizado, placebo-controlado com vinte e uma voluntárias alocadas por conveniência em dois grupos. Os pesquisadores concluíram que após a prática do MP no grupo Pilates houve melhorias na atividade física de lazer, mobilidade toracoabdominal, força muscular respiratória e redução da circunferência da cintura. Já no grupo controle, onde as voluntárias não realizaram o programa de exercícios não houve alterações relevantes nas mesmas variáveis investigadas pelos pesquisadores.

\section{MP e dores lombares}

A dor lombar ou lombalgia é definida como dor ou desconforto que ocorre abaixo da margem costal e acima da margem inferior das dobras glúteas e é um problema de saúde muito comum. A dor lombar é classificada em três categorias: aguda, subaguda e crônica persistente Gaines C (2018).

Para Ferreira TN, et al. (2016), a lombalgia pode causar incapacidade funcional, dependendo se é aguda ou crônica ou dependendo de sua gravidade. Os autores constataram, que o tratamento com exercícios específicos de Pilates apresentam benefícios para pacientes acometidos de lombalgia, com ganho na flexibilidade e força muscular geral. E, com melhoria na postura, da consciência corporal, da respiração, do equilíbrio, da coordenação motora, resultando no relaxamento muscular.

Para Ferreira GQ e Tenório CA (2015), o MP mostrou-se eficaz no tratamento da lombalgia, devido trabalhar com a diminuição da dor; melhorar as alterações musculoesqueléticas; diminuir a tensão muscular, o estresse; melhorar a respiração, além de trabalhar a descompressão das vertebras com seus exercícios específicos utilizados para cada paciente, após a realização da avaliação fisioterapêutica detalhada.

Dessa forma, a técnica de exercícios tem como característica a exigência de músculos abdominais, músculos estabilizadores da coluna vertebral e do assoalho pélvico, que favorece o ganho de forças, 
mantendo-o íntegro, que proporciona melhora nas funções do sistema postural, neuromuscular, reequilíbrio de musculatura e diminuição da dor lombar, constado em estudo com bailarinos (SOUZA AML, 2017).

Assim, quando se trata de prevenção de desconfortos musculoesqueléticos, como dores lombares em gestantes, o MP contribui com o fortalecimento e condicionamento muscular, permitindo melhor adaptação do organismo materno às alterações posturais provenientes da evolução gestacional (NASCIMENTO SL, et al., 2014).

\section{MP e Dores Articulares}

As dores articulares, agudas ou crônicas, estão entre os tipos mais frequentes de dor. As principais causas são o traumatismo e a inflamação, Tais dores, estão associadas ao comprometimento da função articular que pode ser uma simples restrição dos movimentos até sua incapacidade completa. É durante o exercício ou das atividades cotidianas, que esse tipo de dor, tende a se manifestar com maior frequência (SBED, 2010).

O estudo de Antunes MD, et al. (2019) enfocando as dores articulares, foi realizado com dez cuidadores independente do sexo, com a faixa etária de 40 a 65 anos. Os cuidadores receberam orientações posturais gerais relacionadas ao posicionamento correto para exercer atividades de vida diárias (AVDs) e foram submetidos a dez sessões com o método Pilates na bola suíça.

Os autores concluíram que MP e as orientações posturais foram efetivas na redução na dor e sobrecarga dos cuidadores de pacientes neurológicos. Quanto à qualidade de vida os resultados mais favoráveis foram obtidos nos domínios da capacidade funcional, limitação por aspectos físicos, estado geral da saúde e saúde mental.

Deste modo, Escamilla RF (2010) afirma que o exercício de Pilates com uma bola suíça difere de outros exercícios resistidos, porque recruta os músculos responsáveis pela estabilização da coluna vertebral durante o movimento e tendem a melhorar a capacidade funcional, equilíbrio e a propriocepção.

No estudo de revisão de Osterno MCR e Cunha FMAM (2018) que abordou método de Pilates aplicado a artrose, foi constatado que as variáveis equilíbrio e risco de queda foram as mais pesquisadas e os estudos encontraram resultados positivos para as variáveis percentual de gordura, resistência muscular, marcha, e qualidade de vida. Dessa forma, o MP tem se confirmado com uma técnica de confiabilidade e eficiência no tratamento da Artrose, que é uma patologia crônica e multifatorial da lesão osteoarticular e favorece o fortalecimento e colabora para um menor desgaste do joelho.

\section{MP e Capacidades Físicas}

O ser humano possui qualidades físicas próprias que correspondem à parte física corporal do movimento, chamadas de capacidades físicas. Essas capacidades integram um conjunto das possibilidades motrizes naturais e adquiridas, mediante as quais se podem fazer esforços distintos, como resistência, força, velocidade, mobilidade e resistência e flexibilidade (GUTIÉRREZ FG, 2011).

A variável flexibilidade para Coimbra CMS e Coimbra MGF (2019), é frequentemente escolhida por pesquisadores. No entanto, não é tão simples de ser compreendida, ainda mais quando aplicada a uma parcela da população como os idosos, por exemplo.

Apesar do número reduzido de pesquisas, segundo os autores pode-se afirmar, a partir dos trabalhos já publicados, que os benefícios do MP para a flexibilidade nos idosos, contribuem para uma melhor mobilidade, força e equilíbrio, critérios indispensáveis para uma autonomia e melhor qualidade de vida.

Com base na revisão de Torcinelli DL, et al. (2019) para verificar se há influencia do MP sobre a força muscular respiratória, expansibilidade torácica e pico de fluxo expiratório em indivíduos tabagistas, a conclusão foi que o MP pode promover aumento da pressão inspiratória máxima e do pico de fluxo expiratório. Porém, não há influência do método sobre a amplitude de movimento da caixa torácica. Para os autores, há uma carência da literatura em pesquisas demonstrando o efeito do método Pilates sobre os parâmetros respiratórios. 
No estudo de Poletto R e Toigo AM (2017), que abordou os efeitos da prática do MP por atletas de diferentes modalidades. Os resultados mostraram benefícios para o desempenho esportivo, coordenação, flexibilidade, força muscular e postura. Também como alternativa para prevenção de lesões em decorrência da diminuição do comprimento muscular, na flexibilidade, agilidade e equilíbrio estático, na capacidade muscular das pernas dos atletas. Os autores concluíram que de acordo com as pesquisas já realizadas, o MP é uma alternativa eficaz na prevenção e recuperação de lesões desencadeadas pela diminuição do comprimento muscular desta população.

E, Moreira F e Soares JC (2017) avaliou os benefícios do MP em indivíduos com hipercifose na coluna torácica. Os resultados mostraram benefícios variados para os pacientes com hipercifose, quando aplicado de acordo com os princípios do Pilates e que em geral o MP tem sido satisfatório para o tratamento dos pacientes com hipercifose e eficaz no ganho da flexibilidade e no fortalecimento da musculatura abdominal e no reequilíbrio muscular. Os autores recomendam mais pesquisa sobre $o$ assunto.

Muitos idosos têm procurado o método Pilates em busca de saúde, manutenção, prevenção e tratamento para as suas aptidões físicas. Pesquisas demonstraram que o método melhora a marcha nos parâmetros velocidade, ciclo e comprimento do passo e consequentemente redução nos riscos de desequilíbrios e quedas. A melhora da flexibilidade também é consenso entre os pesquisadores (COSTA LMR, et al., 2016).

\section{MP e Estados de Humor}

O humor é compreendido como um estado psicológico, que emanam sentimentos positivos e negativos. Esses sentimentos variam em intensidade, duração que os tornam um indicador do bem estar ou não. Para o estado de humor não se tem um consenso quanto à sua definição (KENNEDY MM e NEWTON M, 1997). Para Evangelista AL, et al. (2014), o MP proporciona benefícios não só físicos, mas também psicológicos. As pessoas foram submetidas a uma sessão de treinamentos com o MP com sete tipos de exercícios de Pilates em sessão de quarenta minutos. Foram analisados os estados de humor antes e depois da sessão com o teste Profile of Mood States (POMS) que analisa seis estados de humor, quais sejam: tensão, depressão, raiva, vigor, fadiga e confusão. Os resultados evidenciaram que o MP pode gerar reduções significativas para os sentimentos de tensão, vigor, raiva e confusão em indivíduos fisicamente ativos.

Os estudos sobre a relação entre exercícios e humor não são recentes. Para Kennedy MM e Newton M (1997) os exercícios mais intensos, tendem a ser eficientes na promoção de alterações positivas nos estados de humor do que os de menor intensidade. Nesse sentido, Werneck FZ, et al. (2010) entende que a intensidade e o tipo do exercício realizado estão relacionados diretamente no estado de humor, após a sessão de exercícios realizados.

Fleming KM, at al. (2020) examinou os efeitos agudos nos estados de humor de oitenta e sete jovens e adultos do sexo masculino, sem experiência anterior com o MP. As variáveis pesquisadas foram, estados de ansiedade, preocupação, sentimentos de tensão, humor deprimido, raiva, energia, fadiga e perturbação total do humor em sessões de trinta minutos de Pilates com quatro repetições de cada movimento sequenciados para iniciantes por quatro dias. Após as análises estatísticas, os resultados mostraram os efeitos positivos do Pilates nos estados de humor dos pesquisados.

\section{CONSIDERAÇÕES FINAIS}

O Pilates é um método utilizado como forma de tratamento por fisioterapeutas e dependendo do objetivo, tem mostrado resultados positivos para diversas patologias. A técnica vem sendo utilizada não só para tratamento de patologias osteomioarticulares, mas também em síndromes metabólicas, doenças neurológicas, estados de humor e os resultados tem sido significativos para hipertensos, na melhora no controle de glicemia de diabéticos e na correção postural, função física e na redução da fadiga de pacientes com esclerose múltipla. E tem sido eficaz na redução de dores musculares, capacidade funcional, qualidade do sono e no tratamento da lombalgia com melhorias da postura e reequilíbrio da musculatura. Todavia, há necessidade de mais estudos e protocolos definidos para confirmar os efeitos do MP em pacientes de fisioterapia relacionados a doenças metabólicas, neurológicas, osteomioarticulares e estados de humor. 


\section{REFERÊNCIAS}

1. ANTUNES MD, et al., Benefícios do método Pilates associado a educação em saúde na sobrecarga e qualidade de vida de cuidadores de pacientes neurológicos. Revista Rede de Cuidados em Saúde, 2019; 13(1): 1-8.

2. AGUIAR SD, et al., Benefício do Método Pilates em mulheres com fibromialgia. ConScientiae Saúde, 2016; 15(3): 440-447.

3. ASSOCIAÇÃO AMERICANA DE DIABETES (ADA). Padrões de atendimento médico em diabetes - 2009. Cuidados com o diabetes. 2009; 32 Suppl 1:S 13-61.

4. ASSOCIAÇÃO BRASILEIRA DE ESCLEROSE MÚLTIPLA - ABEM. O que é Esclerose Múltipla?, 24 out. 2016.

5. COIMBRA CMS e COIMBRA MGR. O método Pilates e a flexibilidade em idosos: revisão de literatura. Brazilian Journal of Development, 2019; 5(10): 21938-21943.

6. COSTA JCB, et al., Os benefícios do método pilates no tratamento da sintomatologia da fibromialgia. Revista Ciência Atual, 2020; 15(1): 138-159.

7. COSTA LPP, et al., Respostas hemodinâmicas do método Pilates em hipertensos: uma revisão sistemática. Revista Brasileira de Ciência e Movimento, 2019; 27(4): 219-227.

8. CORDEIRO AL, et al., Efeito do método de Pilates em pacientes com esclerose múltipla: uma revisão sistemática. Revista Pesquisa em Fisioterapia, 2020; 10(1): 111-117.

9. COSTA LMR, et al., Os efeitos do método pilates aplicado a população idosa: uma revisão integrativa. Revista Brasileira de Geriatria e Gerontologia, 2016; 19(4): 695-702.

10. CRUZ GL, et al. Benefícios do método Pilates nos sintomas da fibromialgia. Revista Faipe, 2018; 8(1): 49-59.

11. DUFF WRD, et al., Impacto do exercício de Pilates na esclerose múltipla: um estudo controlado randomizado. International journal of MS care, 2018; 20(2): 92-100.

12. ESCAMILLA R, et al., Ativação do núcleo muscular durante a bola suíça e exercícios abdominais tradicionais. jornal de fisioterapia ortopédica e esportiva, 2010; 40(5): 265-276.

13. EVANGELISTA AL, et al., A influência do método pilates nos estados de humor em indivíduos fisicamente ativos. Revista Fisioterapia Brasil, 2014; 15(3): 184-188.

14. FERREIRA TN, et al., O método Pilates em pacientes com lombalgia. Revista Saúde \& Ciência em Ação, 2016; 2(1): 55-65.

15. FERREIRA GQ, TENORIO CA. Eficácia do método pilates para dor da região lombar. Revista Visão Universitária, 2015; 1(3): 146-161.

16. FLEMING KM, et al., Efeitos agudos do Pilates nos estados de humor de jovens adultos do sexo masculino. Terapias complementares em medicina, 2020; 49: 102313.

17. GAINES C. Pilates para lombalgia. Pesquisa em enfermagem e saúde, 2018; 41(5): 489-490.

18. GUTIÉRREZ FG. Conceptos y clasificación de las capacidades físicas. Cuerpo, Cultura y Movimiento, 2011; 1(1): 77-86.

19. GUCLU-GUNDUZ A, et al., Os efeitos do pilates no equilíbrio, mobilidade e força em pacientes com esclerose múltipla. Neuro Rehabilitation, 2014; 34(2): 337-342.

20. JESUS LT, et al. Efeitos do método Pilates sobre a função pulmonar, a mobilidade toracoabdominal e a força muscular respiratória: ensaio clínico não randomizado, placebo-controlado. Fisioterapia e Pesquisa, 2015; 22(3): 213-222.

21. JUNGES S, et al., Efeito do método Pilates em fatores de risco para doenças cardiometabólicas: uma revisão sistemática. Scientia Medica, 2015; 25(1): 3-8.

22. KENNEDY MM, NEWTON M. Effect of exercise intensity on mood in step aerobics. The Journal of sports medicine and physical fitness, 1997; 37(3): 200-204.

23. LIPOSCKI DB, et al., Utilização do Método Pilates: reabilitação e condicionamento físico. Revista Fisioterapia Brasil, 2016; 17(1): 56-58.

24. MOREIRA F, SOARES JC. Os benefícios do método Pilates em pacientes com hipercifose. Revista Uningá, 2017; 52(1): 108-113.

25. NASCIMENTO SL, et al., Recomendações para a prática de exercício físico na gravidez: uma revisão crítica da literatura. Revista Brasileira de Ginecologia e Obstetrícia, 2014; 16(9): 423-431.

26. OSTERNO MCR, CUNHA FMAM. Os benefícios do Pilates no tratamento da artrose: uma revisão integrativa. Revista Fisioterapia Ser, 2018; 13(2): 220-223.

27. POLETTO R, TOIGO AM. Efeitos da prática do método Pilates em atletas de diferentes modalidades. Revista Saúde e Desenvolvimento Humano, 2017; 5(2): 59-65.

28. PILATES JP. A obra completa de Joseph Pilates. Sua saúde e O retorno à vida pela Contrologia - São Paulo: Phorte, 2010.

29. SANTOS SN, et al., Os benefícios do exercício físico em indivíduos portadores de diabetes mellitus tipo II. Revista Científica do Centro Universitário de Jales, 2017, 8(1): 1- 13.

30. SOCIEDADE BRASILEIRA DE ENDOCRINOLOGIA E METABOLOGIA-SBEM. A Síndrome Metabólica, 2007.

31. SOCIEDADE BRASILEIRA PARA O ESTUDO DA DOR - SBED. Ano mundial contra a dor musculoesquelética. Artralgia, São Paulo, 2010.

32. SMITH HS, et al., Fibromyalgia: an afferent processing disorder leading to a complex pain generalized syndrome. Pain physician, 2011; 14(2): E217-E245. 
33. SOUZA AML, et al., A eficácia do método pilates na dor lombar em bailarinos com desequilíbrio muscular. Revista Científica do Centro Universitário de Jales, 2017. 8(1): 19-27.

34. SULLIVAN SB, SCHMITZ TJ. Fisioterapia: avaliação e tratamento. 4.ed. São Paulo: Ed. Manole, 2004.

35. SILVA RBF, et al., Método Pilates: Benefícios e aplicabilidade para melhorar a qualidade de vida. Revista Fisioterapia Brasil, 2019; 20(2): 249-262.

36. TORCINELLI DL, et al., Efeito do método Pilates sobre a força muscular respiratória, amplitude de movimento da caixa torácica e pico de fluxo expiratório em tabagistas. Revista Fisioterapia Brasil, 2019. 20(4): S9-S14.

37. VAZ RA, et al., O Método Pilates na melhora da flexibilidade: revisão sistemática. Revista Brasileira de Prescrição e Fisiologia do Exercício, 2012; 6(31): 25-31.

38. WELLS C, et al., Pilates exercise: a systematic review. Complementary therapies in medicine, 2012; 20(4): 253262.

39. WERNECK, FZ et al., Efeito agudo do tipo e da intensidade do exercício sobre os estados de humor. Revista Brasileira de Atividade Física \& Saúde, 2010; 15(4): 211-217. 